\title{
Analgesia and Sedation in Intensive Care Unit
}

\author{
Rioko Kimiko Sakata, TSA ${ }^{1}$
}

Summary: Sakata RK - Analgesia and Sedation in Intensive Care Unit.

Background and objectives: Many Intensive Care Unit (ICU) patients present pain. The administration of analgesics and sedatives is crucial for patient comfort and to reduce stress, as well as to prevent delay in recovery and ventilator weaning. The objective of the present study was to conduct a review on analgesia and sedation in the ICU.

Content: The present study reviewed the causes of pain, the methods used to evaluate the intensity of pain and sedation and the conducts employed in pain treatment and sedation. Drug selection is important in order to prevent excessive sedation, by performing analgesia before the sedation.

Conclusions: The most commonly employed drugs are morphine, fentanyl, midazolam and propofol. Other medications are less frequently used.

Keywords: ANALGESIA; SEDATION; INTENSIVE THERAPY.

\section{INTRODUCTION}

Most patients at the Intensive Care Unit (ICU) experience pain, fear and anxiety ${ }^{1,2}$. The administration of analgesics and sedatives is crucial for patient comfort and to reduce stress, as well as to prevent delay in recovery and ventilator weaning1.

One study showed that less than $50 \%$ of the patients have adequate pain control at the ICU ${ }^{3}$. The barriers were: the physician's conduct, use of protocols without evidence, the professionals' resistance in changing the conduct, inadequate method of assessment and insufficient training of the professionals regarding pain assessment and treatment.

Pain relief is essential for the patient's adequate recovery $1,2,4,5$. The benefit is more evident in the patient that presents alterations in several organs when the pain causes more severe alterations.

Recovery is significantly influenced by the choice of analgesic and sedative agents, deficient or excessive sedation and the insufficient control of pain ${ }^{2}$.

In spite of published guidelines and studies, the use of strategies that have been approved at the routine can occur slowly ${ }^{2}$. Analgesia and sedation at the ICU are complex due to several comorbidities, drug interactions and organ dysfunctions ${ }^{6}$.

Received from the Service of Pain Management - Department of Anesthesiology, Pain and Intensive Care of Escola Paulista de Medicina da Universidade Federal de São Paulo (EPM/ UNIFESP), SP.

1. Associate Professor and Coordinator of the Department of Pain and Intensive Care of EPM/UNIFESP

Submitted on March 22, 2010

Accepted on June 4, 2010.

Correspondence to:

Dra. Rioko Kimiko Sakata

Rua Três de Maio, 61

Vila Clementino

04044-020 - São Paulo, SP

E-mail: riokoks.dcir@epm.br

\section{Critically-ill patients}

Critically-ill patients present important alterations in the pharmacology of analgesics. Very often it is not possible to administer drugs orally. The absorption of medication administered orally is unpredictable in a patient with gastrointestinal function alterations and decreased splanchnic blood flow.

There is a decrease in the hepatic blood flow and drug clearance in arterial hypotension, in trauma, in sepsis, in hipoxemia and when some drugs are being administered.

Organ dysfunction causes alteration in the clearance of drugs and metabolites ${ }^{7-10}$. In kidney failure there is accumulation of analgesics, sedatives and active metabolites normally eliminated by the kidneys. In shock, drug clearance is slower 9,10 .

In the patient with hypoproteinemia, more free drugs are associated with a higher possibility of toxicity. Acidemia also causes increase in the active drug levels. Alterations in the hematoencephalic barrier also facilitate the passage of some drugs.

\section{What causes pain?}

Many ICU patients experience pain. The causes are ${ }^{7,11,12}$ :

- Tracheal aspiration

- Change of decubitus

- Dressing wounds

- Tracheal tube

- Trauma

- Surgical lesion

- Burns

- Nasogastric tube

- Central catheter

- Arterial Catheter

- Drains

- Pressure ulcers

- Venous or arterial puncture

- Bandages for limb restraining 


\section{Pain and sedation assessment}

Less than $50 \%$ of the professionals evaluate the pain ${ }^{7}$. Pain and sedation assessment is particularly complex in an ICU, as the patients are very often incapable or unable to verbally communicate with the professionals $4,11,13-15$. The patient cannot establish communication with the professionals due to several reasons: tracheal intubation, alteration of consciousness, sedation, and effect of medications ${ }^{11}$.

The patient undergoing mechanical ventilation must be assessed regarding pain and sedation to have the drug dose optimized. That reduces the need for ventilation and ICU stay duration ${ }^{6}$.

Scales help to detect pain ${ }^{7}$. A patient that can communicate verbally can be evaluated through the numerical verbal scale (0 to 10) ${ }^{1}$, which requires a higher degree of comprehension than the 4-point verbal rating scale (VRS-4), in which the pain can be absent $=1$, mild $=2$, moderate $=3$ or severe $=4{ }^{18}$. The correspondence of the descriptive scale with the numerical one would be: absent pain $=0$; mild $=1-3$; moderate $=4-6$ and severe $=7-10^{14}$. Scores $>3$ in the numerical scale or 2 at the verbal scale are unacceptable ${ }^{14}$.

Pain causes sympathetic stimulation accompanied by tachycardia and increased blood pressure, symptoms that can help to detect pain. However, these alterations have little specificity at the ICU and can be caused by vasopressors, betaadrenergic blockers, anti-arrhythmic drugs, sedatives and pathological conditions (sepsis, shock, hypoxemia and fear) ${ }^{11}$.

When the patient is unable to communicate, other methods must be used ${ }^{7-14}$. The Behavioral Pain Scale (BPS) can be used, with scores from 3 to 12 , through the observation of the facial expression, body movements, muscle tension and synchrony with the ventilator ${ }^{1-2}$.

The BPS, which is easy to interpret, is used to assess the pain in sedated patients or those submitted to mechanical ventilation (Chart I). If the score is $>6$, it is considered unacceptable ${ }^{14}$.

The facial expression is the item that contributes the most to pain assessment, followed by limb movement and acceptance of ventilation ${ }^{11}$. The following are pain behaviors: gri-

Chart I - The Behavioral Pain Scale Analyzes:

Facial expression

Relaxed: 1

Partially tense: 2

Totally tense: 3

Grimace: 4

Movements of upper limbs

Relaxed: 1

Partially flexed: 2

Totally flexed: 3

Totally contracted: 4

Mechanical ventilation

Tolerating movements: 1

Coughing, but tolerating during most of the time: 2

Fighting the ventilator: 3

Impossible to control the ventilator: 4 macing, frowning, rigidity, tightly closed eyelids, wrinkling the nose, elevated upper lip, verbalization, and closed fists ${ }^{11}$. The acceptance of the mechanical ventilation can be affected by hypoxemia, bronchospasm and secretion ${ }^{11}$.

The scale of sedation was described by Ramsay (Chart II) ${ }^{11,14}$. When the scale is used there is a lower incidence of excessive sedation ${ }^{1}$. Approximately $70 \%$ of the professionals use scales for sedation ${ }^{16}$.

\section{Chart II - Ramsay Score}

- anxious, agitated

2 - cooperative, oriented and serene

3 - sleeping, drowsy and responding easily to commands

4 - sleeping and responding to stimuli on glabella

5 - sleeping and responding slowly to pressure on glabella

6 - sleeping without response to pressure on glabella

The desirable characteristics of a scale are:

- Easy to apply

- Easy to interpret

- Possibility to evaluate small alterations

- Evaluation of sedation for drug titration

- Evaluation of agitation

\section{Treatment of pain and sedation}

The methods of analgesia can act on different points of the pain pathways, reducing the activation of nociceptors or the passage of the stimulus, activating the inhibitory pathways and altering pain perception.

To alleviate pain and anxiety, analgesic and sedative agents are administered ${ }^{12}$. A study disclosed the different protocols adopted for analgesia and sedation in the ICU 1 . There is a discrepancy between the need for sedation and the way it is carried out ${ }^{16}$. Opioids, benzodiazepines and propofol are the basic medications used to give the patient comfort and facilitate mechanical ventilation. Most professionals use opioids associated with GABA agonists ${ }^{1}$.

No medication has all the characteristics of the ideal analgesic or sedative. To optimize pain treatment, the physician must know the pharmacokinetic and pharmacodynamic differences that can affect the safety and effectiveness of analgesics and sedatives ${ }^{17}$. Modifications in the pharmacokinetics and pharmacodynamics with long-term administration, alterations in the protein binding and volemia and organ dysfunction cause variable results ${ }^{1}$. Pain treatment requires knowledge of the action mechanisms, pharmacokinetics, latency and the duration of the analgesia and the side effects. The correct analgesic agents must be administered at the adequate dose to a certain patient at the right moment ${ }^{1}$. It is important to know what clinical alterations the patient presents and the medications he or she uses, as it is possible that there will be an interaction with the analgesics.

The sedation procedures for ICU patients have evolved and depend on the patient and the available drug ${ }^{7}$. The ideal level 
of sedation is controversial. In the past, deep sedation was preferred; however, the recovery is influenced by the choice of the agent, by insufficient or excessive sedation and by the inadequate control of pain ${ }^{2}$.

Inadequate sedation results in pain, anxiety, agitation, auto-extubation, catheter removal, myocardial ischemia and hypoxemia ${ }^{1}$. Excessive or prolonged sedation causes compression ulcers, nerve compression, delirium and prolonged mechanical ventilation ${ }^{1}$.

\section{Protocols, algorithms and guidelines}

The guidelines, protocols and algorithms can promote conduct based on evidence, decreasing the variation in clinical practice and the possibility of excessive or prolonged sedation ${ }^{1}$. The first guideline for sedation in ICU dates from $1995{ }^{7}$.

Approximately $50 \%$ of the professionals adopt protocols for analgesic and sedative agent administration ${ }^{16}$. The choice varies according to the place and type of ICU ${ }^{2}$. The protocols promote a more adequate sedation, less pain, decrease in the variation between patients and the amount of drugs, as well as time of mechanical ventilation and ICU stay duration ${ }^{1,7}$.

Analgesia and sedation must be carried out with increase or decrease and the change in the medication, according to the specific target. A daily interruption in the sedative and opioid infusion can also be carried out ${ }^{1}$. Drug and metabolite accumulation must be avoided, as it can delay recovery ${ }^{1}$.

The algorithms must have specific objectives and targets, with the adoption of effective and applicable protocols, in order to evaluate analgesia, agitation and sedation, as well as incorporate the selection of medication ${ }^{1}$.

\section{Techniques of analgesia and sedation}

Sedation techniques have evolved considerably with the emergence of new medications and treatments based on evidence $^{7}$. Drug titration through the use of scales represented an improvement, with the maintenance of mild sedation. Excessive sedation results in a prolonged recovery, but pain and anxiety relief must be adequate 1 .

The important characteristics of analgesic drugs and techniques are $2,11,12,17$ :

- Evidence of effectiveness

- Rapid action and recovery

- Predictability between dose and response

- Absence of accumulation and toxicity

- Absence of interaction

- Indication for critical patients

- Easy titration and monitoring

- Tolerable adverse effects

- Low cost

\section{Analgesia technique before sedation}

Measures to improve recovery at the ICU include analgesia before sedation and recognition of the adverse effects associated with sedative medications ${ }^{18}$.

In the technique called "analgesia first" or "A1", the drugs used for sedation are administered only after the use of analgesics ${ }^{7}$. The patients that received "A1" obtain comfort and less than $50 \%$ of them need sedation ${ }^{7}$. Analgesia before sedation can reduce the need for sedatives and the time of mechanical ventilation duration ${ }^{7}$.

\section{Administration in bolus and infusion}

The administration in bolus is associated with the variation in the plasma concentration with peaks and valleys, excessive sedation and anxiety. The infusion maintains a more stable concentration, but can prolong the time until the patient wakes up, due to the accumulation of the drug ${ }^{2}$.

\section{Daily Interruption of sedation (DIS)}

Individualized sedation, with the adoption of specific protocols and questionnaires and the daily interruption can improve the outcome ${ }^{2}$. Daily Interruption of Sedation (DIS) has been incorporated by around $30 \%$ of the ICUs 7 .

The concept of daily interruption of the infusion appeared to avoid excessive sedation, in order to evaluate the need for sedatives and decrease the accumulation, time of mechanical ventilation and ICU stay duration 1,2 .

Approximately 10 years ago protocols started to be used, which included the temporary interruption of analgesic and sedative agents until the patient could give 3-4 simple answers or present agitation ${ }^{1}$. That decreases the time of mechanical ventilation and ICU stay duration, as well as the need for complementary examinations ${ }^{1,2}$. After the patient wakes up, the infusion is re-established in a titrated form with the previous dose or half the previous dose 1,12 .

The main reasons not to perform DIS are: fear of respiratory impairment, lack of acceptance by the nursing staff, fear of catheter removal and cardiovascular, respiratory or neurologic instability 2,12 . Some professionals prefer not to interrupt sedation, fearing the increase in the intracranial pressure and the generation of ventilatory difficulties and hemodynamic instability ${ }^{12}$. DIS is associated with a faster resolution of respiratory failure due to several factors. There is a decrease in the accumulation of sedatives and metabolites with faster recovery of the mental state for effective ventilation ${ }^{1}$. There is also an additional opportunity for the physician to recognize that the patient is capable of breathing ${ }^{1}$. A decrease in the dose of GABA agonist and opioids is also observed ${ }^{1}$. There is a decrease in the rate of complications due to the shorter time of mechanical ventilation ${ }^{1}$. There is evidence that DIS is safe to prevent excessive sedation and facilitate the recovery of respiratory failure ${ }^{1}$. 
The problems associated with DIS are: increase in auto-extubation, removal of monitoring apparatus, anxiety, intracranial pressure worsening, abstinence manifestations ${ }^{1,6}$. It is necessary to observe the patient carefully in order to prevent autoextubation and other consequences of agitation when DIS is used ${ }^{1}$. There is an increase in heart rate (HR) and systemic blood pressure and catecholamine levels ${ }^{1}$. Further studies are necessary to evaluate its effect on a patient with hypertensive emergency and myocardial infarction ${ }^{1}$. The sudden awakening might be associated with psychological stress ${ }^{1}$. The repeated withdrawal can result in episodes of drug and alcohol abstinence manifestations in high-risk patients ${ }^{1}$. These manifestations occur 6 hours after the withdrawal of ultra-short acting drugs, such as sufentanyl, midazolam and propofol. For drug and alcohol-dependent individuals, the withdrawal is potentially deleterious and an alternative must be used ${ }^{1}$.

DIS must not be used in some situations, such as ${ }^{1}$ :

- The patient is receiving sedatives for control of seizures or alcohol abstinence

- When the sedative dose for the control of agitation is being increased

- The patient is receiving neuromuscular blockers

- There is evidence of previous myocardial infarction (24 hours before)

- There is evidence of intracranial pressure increase

\section{Medications and techniques for analgesia and sedation}

\section{Anti-inflammatory agents, Paracetamol and Dipyrone}

The anti-inflammatory agents present a significant proteinbinding capacity, with increase of side effects in patients with hypoalbuminemia. They are rarely indicated in the ICU, as they can precipitate kidney failure ${ }^{19-21}$. The predisposing factors are: previous renal alteration, hypovolemia, arterial hypotension, arterial hypertension, older age, ascites, congestive heart failure, liver cirrhosis and concomitant use of medications that lead to kidney injury 10,19,22. The gastrointestinal effects are also more frequent in the ICU. The gastrointestinal risk factors are: concomitant use of multiple anti-inflammatory agents, history of ulcer or digestive complaint, previous history of upper digestive tract bleeding, presence of cardiovascular diseases, concomitant use of corticosteroids or anticoagulants and older age. Paracetamol can be used at a dose of 1g. $6 \mathrm{~h}^{-114}$. Dipyrone can be used at a dose of $1 \mathrm{~g} \cdot 4 \mathrm{~h}^{-1}$.

\section{Opioids}

The most commonly used analgesic agents are morphine and fentanyl and the preference for one of them varies from country to country ${ }^{2,12}$. Other opioids used are remifentanil, Sufentanil and codeine ${ }^{14,18}$. There are no data to support the preference of analgesic over the other. There development of fast opioid tolerance can occur fast and then there is the need to increase the dose, which facilitates the occurrence of opioid-induced hyperalgesia ${ }^{18}$.
Morphine can be administered subcutaneously at a dose of 5-10 mg.4-6h-1 or as intravenous infusion at a dose of 30-50 mg. $\mathrm{d}^{-1}{ }^{14,18}$. For procedure, a bolus of $5-10 \mathrm{mg}$ de morphine can be added ${ }^{14}$. This opioid forms an active metabolite and presents unpredictable kinetics in patients with organ dysfunction ${ }^{11}$. In liver failure, there is a decrease in the metabolism of morphine, whereas in kidney failure there is an accumulation of the metabolite (Morphine-6-glucuronide-M6G) ${ }^{8-10}$. For patients in shock, the elimination is slower ${ }^{2,6}$. The risk of respiratory depression is higher in newborns, in patients with cognitive alteration, those who are hemodynamically unstable, with history of apnea and respiratory disease. Morphine can cause arterial hypotension ${ }^{11}$.

Fentanyl is administered via IV route at a dose of $300-700$ $\mu \mathrm{g} \cdot \mathrm{h}^{-1}{ }^{18}$. Its metabolite is inactive and excreted by urine and bile. Fentanyl does not cause hemodynamic alteration and it is indicated for patients with cardiovascular impairment. In patients with kidney failure, it is more indicated than morphine ${ }^{1}$.

Remifentanil is a fast-acting drug and presents an equally fast recovery ${ }^{23}$. Its metabolism does not depend on the liver. It can be used in patients that need frequent neurological assessment ${ }^{7}$. The dose used is $6-60 \mu \mathrm{g} \cdot \mathrm{kg}^{-1} \cdot \mathrm{h}^{-1} 24$.

Codeine is a weak opioid that acts after being transformed into morphine. Methadone has a long half-life and accumulation can occur with continuing administration. Meperidine must not be used as it is metabolized and forms Normeperidine, which causes seizures. In liver and kidney dysfunction, there is an increase in the time of drug elimination ${ }^{9,10}$. Meperidine has a negative inotropic effect, presents anticholinergic activity and can cause tachycardia.

\section{Tramadol}

Tramadol is the synthetic analog of codeine, of which analgesic effect occurs through its action on mu receptors and inhibition of serotonin and noradrenalin reuptake.

\section{Patient-controlled analgesia (PCA)}

PCA is a technique adopted to reduce the interval between the complaint of pain and its relief. It can be used in patients that are capable of handling the apparatus that releases the opioid. The medication used is usually morphine via intravenous route and fentanyl via epidural route ${ }^{2}$.

\section{GABA (gamma-aminobutyric acid) agonists}

GABA agonists used are the benzodiazepines (midazolam, lorazepam and flunitrazepam) and propofol ${ }^{1}$. Midazolam, lorazepam and propofol are the medications more frequently used for sedation in the ICU 7,12,14. The choice of one of these drugs varies from country to country ${ }^{2}$. Sedation with midazolam and propofol was an innovation to prevent prolonged sedation 7 .

Midazolam is more titratable ${ }^{12}$. Its action is fast and the duration is short, with one dose. It is medically indicated for short-term sedation 2. Prolonged administration results in accumulation of the drug and the active metabolite (alpha-hydroxymidazolam), especially in obese patients, those presenting low concentrations 
of albumin or kidney failure $7,11,18$. Some medications that use the same enzyme for metabolism (fentanyl and propofol) increase the duration ${ }^{7}$. When used at a dose of $25 \mathrm{mg} \cdot \mathrm{h}^{-1} 25$ midazolam can cause arterial hypotension ${ }^{11}$.

Lorazepam is seldom used in Europe and very often used in the United States ${ }^{2}$ and presents an advantage in relation to long-term diazepam ${ }^{7}$. This medication more frequently results in excessive sedation than midazolam ${ }^{12}$. It is indicated for longterm sedation $(>24 \mathrm{~h})^{2}$. When administered by parenteral route, it can cause toxicity by propylene glycol, mainly with very large doses $\left(15-25 \mathrm{mg} \cdot \mathrm{h}^{-1}\right)^{2,7,18}$. The use of small doses $\left(1 \mathrm{mg} \cdot \mathrm{kg}^{-1} \cdot \mathrm{d}^{-1}\right)$ can cause adverse effects, such as acute kidney injury and metabolic acidosis ${ }^{18}$. The development of tolerance occurs fast with the use of benzodiazepines by intravenous route ${ }^{18}$. Benzodiazepines can cause delirium ${ }^{2,6,7,18}$.

Propofol presents fast action and clearance ${ }^{7}$ and it is indicated for short-term sedation ${ }^{2}$. Propofol can cause arterial hypotension, respiratory depression, hypertriglyceridemia, pancreatitis and propofol infusion syndrome $2,7,18$. The latter is the most severe event and the possible mechanisms include: mitochondrial enzyme inhibition, prevention of fatty-acid oxidation and metabolic alteration of carbohydrates in fatty substrates. There is an increase in triglyceride levels, hypotension, arrhythmia, kidney failure, hyperkalemia, rhabdomyolysis and liver dysfunction ${ }^{7}$. Although associated with the prolonged use of large doses (> $70 \mu \mathrm{g} \cdot \mathrm{kg}^{-1} \cdot \mathrm{min}^{-1}$ ), many reports have mentioned the effect after lower and shorter-term doses ${ }^{7}$.

\section{Alpha-2 adrenergic agonists}

Alpha-2 adrenergic agonists (clonidine and dexmedetomidine) are alternatives for benzodiazepines ${ }^{7}$. Dexmedetomidine promotes the decrease in motor activity, mental stability, allowing better care by the physician, nurse and physical therapist ${ }^{18}$. Its metabolites are inactive and the clearance is urinary and fecal ${ }^{18}$. Analgesia and sedation are related to the binding to central noradrenergic receptors ${ }^{7,18}$. It can modulate the descending inhibition from the locus coeruleus with noradrenaline release ${ }^{18}$. Dexmedetomidine reduces the incidence of delirium and the duration of mechanical ventilation ${ }^{7,18}$. It causes little respiratory depression and it is administered at a dose of $1 \mu \mathrm{g} \cdot \mathrm{kg}^{-1}$, followed by an infusion of $0.1-0.7 \mu \mathrm{g} \cdot \mathrm{kg}^{-1} \cdot \mathrm{h}^{-1}$ for analgesia and sedation, with the dose being titrated ${ }^{18}$. The side effects are hypertension and bradycardia with the initial dose due to alpha-2B receptor stimulation. With the infusion, hypotension occurs due to the central sympatholytic effect and noradrenaline decrease ${ }^{7,18}$. The sympatholytic effect can be beneficial, as it reduces tachycardia and arterial hypertension, or undesirable, as they cause hypotension and bradycardia 7 . Other complications include: cardiac block, sinus arrest, atrial fibrillation ${ }^{25}$. A hypertensive effect can occur after the end of the central alpha-2 agonist action ${ }^{25}$. Clonidine can be used for short and long-term sedation ${ }^{2}$.

\section{Etomidate}

Etomidate was administered by infusion, until it was acknowledged that this drug is not a safe one ${ }^{7}$. Currently, it is not used for sedation.

\section{Neuromuscular blockers}

In the past, they were often used for deep sedation ${ }^{7}$. Subsequently, there were reports of neuromuscular dysfunction after the prolonged administration of neuromuscular blockers ${ }^{7}$. Currently, the indications for the use of neuromuscular blockers are: patient intubation, asynchrony with ventilator, hypercapnia, non-conventional ventilation, increase in intracranial pressure and procedure performance ${ }^{12}$. The most frequently used drugs are: pancuronium, rocuronium, vecuronium and cisatracurium. The factors for drug choice are: the physician's experience, action duration, half-life, clearance mechanisms and the patient's individual factors ${ }^{12}$.

\section{Other drugs}

Other drugs are used as adjuvant analgesic therapy in the ICU: antidepressant and anticonvulsant agents, neuroleptics, neuromuscular blockers are less frequently used in ICU patients.

\section{Regional analgesia}

Opioids, local anesthetics and clonidine are more frequently used for regional analgesia ${ }^{2}$. Opioids are the ones most often used by spinal route. As the medication is injected into the action site, there is no need for it to pass through barriers and small doses of opioids are enough to provide the analgesic effect, particularly with a hydrophilic opioid such as morphine. The effect occurs mainly on $\mathrm{C}$ fibers and at a smaller extent on A-delta fibers with no alterations on autonomic, motor from tact to proprioception. In general, opioids are associated with local anesthetics in order to attain a better analgesia, with a decrease in side effects ${ }^{25}$. As they have different action mechanisms, the association of small doses of the medications has a synergistic effect. Opioids administered by the spinal route are indicated for post-operative analgesia, trauma (costal arch fractures and lower-limb bones), burns and lower-limb ischemia pain. This technique can be used in patients with alterations in several organs. This method provides intense analgesia, with less sedation in comparison to the systemic opioid. The following are frequent side effects when used for acute pain: pruritus, nausea, vomiting and urinary retention.

Principles for analgesia and sedation ${ }^{1}$ :

- Establish targets

- Re-evaluate frequently

- Measure the intensity of pain and of sedation with validated scales

- Select medications based on important characteristics and evidence

- Select safe drugs for the at-risk population

- Avoid excessive sedation

- Control pain and agitation

- Multidisciplinary treatment

- Choose easy-to-use techniques

- Use protocols, algorithms and guidelines 
11. Aïssaoui $Y$, Zeggwagh AA, Zekraoui A et al. - Validation of a behavioral pain scale in critically ill, sedated, and mechanically ventilated patients. Anesth Analg, 2005;101:1470-1476.

12. Mehta S, Burry L, Fischer S et al. - Canadian survey of the use of sedatives, analgesics, and neuromuscular blocking agents in critically ill patients. Crit Care Med, 2006;34:374-380.

13. Gholami B, Haddad WM, Tannenbaum AR - Agitation and pain assessment using digital imaging. Conf Proc IEEE Eng Med Biol Soc, 2009;2176-2179.

14. Ahlers SJ, van der Veen AM, van Dijk M et al. - The use of the Behavioral Pain Scale to assess pain in conscious sedated patients. Anesth Analg, 2010;110:127-133.

15. Schnakers $C$, Chatelle $C$, Vanhaudenhuyse A et al. - The nociception coma scale: a new tool to assess nociception in disorders of consciousness. Pain, 2010;148:215-219.

16. O'Connor M, Bucknall T, Manias E - Sedation management in Australian and New Zealand intensive care units: doctors' and nurses' practices and opinions. Am J Crit Care, 2010;19:285-295.

17. Devlin JW, Roberts RJ - Pharmacology of commonly used analgesics and sedatives in the ICU: benzodiazepines, propofol, and opioids. Crit Care Clin, 2009;25:431-449.

18. Wallace S, Mecklenburg B, Hanling S - Profound reduction in sedation and analgesic requirements using extended dexmedetomidine infusions in a patient with an open abdomen. Mil Med, 2009;174:12281230.

19. Denson DD, Katz JA - Nonsteroidal anti-inflammatory agents, em: Sinatra RS, Hord AH, Ginsberg B et al. - Acute pain. St Louis, MosbyYear, 1992;112-123.

20. Katz JA - Opioids and Nonsteroidal Antiinflammatory Analgesics, em: Raj PP - Pain medicine: a comprehensive review. St Louis, Mosby, 1996;126-141.

21. Breyer MD, Harris RC - Cyclooxygenase 2 and the kidney. Curr Opin Nephrol Hypertens, 2001;10:89-98.

22. Appleton I - Non-steroidal anti-inflammatory drugs and pain, em: Dickenson A, Besson JM - The pharmacology of pain. Library of Congress. Germany 1997;42-54.

23. Battershill AJ, Keating GM - Remifentanil: a review of its analgesic and sedative use in the intensive care unit. Drugs, 2006;66:365-385.

24. Muellejans B, Matthey T, Scholpp $\mathrm{J}$ et al. - Sedation in the intensive care unit with remifentanil/propofol versus midazolam/fentanyl: a randomised, open-label, pharmacoeconomic trial. Crit Care, 2006;10:R91.

25. Scott DA, Blake D, Buckland $M$ et al. - A comparison of epidural ropivacaine infusion alone and in combination with 1,2 , and $4 \mathrm{microg} / \mathrm{ml}$ fentanyl for seventy-two hours of postoperative analgesia after major abdominal surgery. Anesth Analg, 1999, 88:857-864.

01. Sessler CN, Pedram S - Protocolized and target-based sedation and analgesia in the ICU. Crit Care Clin, 2009;25:489-513.

02. Mehta S, McCullagh I, Burry L - Current sedation practices: lessons learned from international surveys. Crit Care Clin, 2009;25:471-488.

03. Byrd PJ, Gonzales I, Parsons V - Exploring barriers to pain management in newborn intensive care units: a pilot survey of NICU nurses. Adv Neonatal Care, 2009;9:299-306.

04. Suominen $\mathrm{H}$, Lundgrén-Laine $\mathrm{H}$, Salanterä $\mathrm{S}$ et al. - Evaluating pain in intensive care. Stud Health Technol Inform, 2009;146:192-196.

05. Sieber FE - Postoperative delirium in the elderly surgical patient. Anesthesiol Clin, 2009;27:451-464.

06. Kumar AB, Brennan TJ - Pain assessment, sedation, and analgesic administration in the intensive care unit. Anesthesiology, 2009;111:1187-1188.

07. Riker RR, Fraser GL - Altering intensive care sedation paradigms to improve patient outcomes. Crit Care Clin, 2009;25:527-538.

08. Stevens DS, Edwards WT - Management of pain in intensive care settings. Surg Clin North Am, 1999;79:371-386.

09. Power BM, Forbes AM, van Heerden PV et al. - Pharmacokinetics of drugs in critically ill adults. Clin Pharmacokinet, 1998;34:25-56.

10. Bodenham A, Shelly MP, Park GR - The altered pharmacokinetics and pharmacodynamics of drugs commonly used in critically ill patients. Clin Pharmacokinet, 1988;14:347-373.

Resumen: Sakata RK - Analgesia y Sedación en Unidad de Cuidados Intensivos.

Justificativa y objetivos: Una gran parte de los pacientes de UCI presenta dolor. La administración de analgésico y sedante es fundamental para la comodidad del paciente, la reducción del estrés, y para evitar el retraso en la recuperación y la liberación de la ventilación mecánica. El objetivo de este estudio fue realizar una revisión sobre analgesia y sedación en UCl.

Contenido: Se realizó una revisión de las causas del dolor, de los métodos para evaluar la intensidad del dolor y de la sedación, y también de las conductas usadas para el tratamiento del dolor y la sedación. Es importante la selección de medicamentos, para evitar la sedación excesiva, con la realización de la analgesia antes de la sedación.

Conclusiones: Los fármacos más utilizados son la morfina, el fentanil, el midazolam y el propofol. Otros medicamentos se usan con menor frecuencia. 